\title{
Das Patientenvertrauen im Fokus
}

\author{
Stefan Büchia, Sebastian Haas ${ }^{b}$, Privatklinik Hohenegg \\ ärztlicher Direktor, Prof. Dr. med., Mitglied FMH \\ b Stv. Ärztlicher Direktor, Dr. med., MHA, Mitglied FMH
}

\begin{abstract}
Im Rahmen einer umfangreichen Neuorganisation der psychiatrischen Privatklinik Hohenegg entwickelten die Autoren das Hohenegg-Kontextmodell, das alle therapeutischen Prozesse konsequent an drei Dimensionen des Patientenvertrauens orientiert: personales Vertrauen - Beziehung, krankheitsbezogenes Vertrauen Expertise und prozessuales Vertrauen - Plausibilität. Die theoretische Entwicklung des Modells wird in diesem ersten Teil des Artikels vorgestellt. In einem zweiten Teil, der nur online verfügbar ist, wird die praktische Implementierung und deren erste wissenschaftliche Evaluation thematisiert ${ }^{1}$.
\end{abstract}

\section{Résumé}

Des résultats d'études actuelles montrent clairement que l'efficacité d'une psychothérapie dépend moins de techniques spécifiques que des trois aspects suivants de la confiance du patient: 1. confiance personnelle - relation, 2. confiance par rapport à la maladie - expertise et 3 . confiance procédurale - plausibilité.

Dans le cadre d'une réorganisation en profondeur de la clinique psychiatrique privée Hohenegg, nous avons mis au point le modèle de contexte Hohenegg, qui adapte systématiquement tous les processus thérapeutiques à ces trois dimensions de la confiance du patient. Le développement théorique du modèle est évoqué dans la première partie de l'article, tandis que la seconde (uniquement en ligne) est consacrée à sa mise en œuvre pratique et à son évaluation scientifique.

\section{Teil 1: Entwicklung des Hohenegg- Kontextmodelles}

\section{Einführung/Ausgangslage}

In den letzten Jahren hat sich der therapeutische Diskurs stationärer Institutionen vornehmlich an den ökonomischen Folgen der Einführung von Fallpauschalen orientiert. Aus unserer Sicht ist es dringend notwendig, zu fachlich-inhaltlichen Diskussionen zurückzukehren und die stationären Behandlungen durch gezielte Orientierung an Kernbedürfnissen und Werten weiter zu verbessern.

1 Der zweite Teil des Artikels ist online einsehbar unter www.saez.ch $\rightarrow$ Aktuelle Ausgabe therapeutische Grundlagen, Therapiemodelle wie auch die aktuellen therapeutischen Angebote initiiert, in welchen die Mehrheit der therapeutisch Tätigen der Klinik intensiv involviert war. Die Umsetzung der Konzepte resp. die Implementierung der neuen Prozesse begann im Januar 2016 und wird im 2. Teil des Artikels vorgestellt.

Unsere Modellentwicklung basiert auf folgenden Grundannahmen:

\section{Menschenbild}

Wir verstehen die Würde des Menschen als unantastbar und unverlierbar. In unserem Verständnis ist jeder Mensch im Innersten Selbstzweck und nicht Mittel zu einem Zweck. Trotz unseres Wissens um die biologische und biographische Bedingtheit eines jeden Menschen gehen wir von einem letzten nicht ergründbaren Geheimnis eines Jeden aus, welches den Menschen ausmacht - seinem Selbst. Die individuelle Entwicklung basiert auf Beziehung. Das Ich wird am Du zum Ich (Buber) [1]. Sie vollzieht sich als ein Prozess, der Krisen beinhaltet. Krisen sind Zeiten des Umbruchs, in denen die Person die Gefahr des Zusammenbruchs erlebt, die gleichzeitig aber auch die Chance für Neues beinhalten.

\section{Leiden und Person}

Im Zentrum all unserer therapeutischen Aktivitäten steht der leidende Mensch. Wir definieren Leiden nach Cassell in folgender Weise: «Leiden ist ein Zustand von schwerem Distress, der im Zusammenhang mit dem drohenden Verlust der Intaktheit der Person steht» [2]. Leiden ist somit eine Dimension, die den Menschen in 
seinem Person-Sein als körperliches, psychisches und soziales Wesen betrifft und wird dann erlebt, wenn ein für die Person relevanter Aspekt bedroht ist.

\section{Gesundheit und Krankheit}

Entsprechend aktuellen Verständnismodellen definieren wir Gesundheit als einen Zustand gelingender flexibler Adaptation an die körperlichen, psychologischen und sozialen Ansprüche des Lebens [3]. Gesundheit und Krankheit sind die zwei beiden entgegengesetzten Pole des Lebens-Kontinuums. Jeder Kranke hat, solange er lebt, gesunde Anteile, jeder Gesunde trägt auch Krankheit in sich [4]. Unsere Therapien versuchen das Individuum nachhaltig, d.h. über den stationären Behandlungszeitraum hinaus zu mehr Flexibilität und Adaptivität ans Leben, d.h. zu mehr Gesundheit zu führen. Dabei versuchen wir, die Beeinträchtigung durch Krankheit entsprechend dem aktuellen Wissenstand zu reduzieren. Zugleich fördern wir gezielt die gesunden Anteile und die persönlichen Ressourcen unserer Patienten.

\section{Umgang mit Begrenzung: Akzeptanz oder "aktive Resignation»}

Die aktuelle Forschung belegt, dass die häufigsten psychischen Krankheiten, die in einer psychiatrischen Klinik wie der Privatklinik Hohenegg behandelt werden (z.B. Depressionen, generalisierte Ängste oder somatoforme Schmerzstörungen) im Langzeitverlauf einen chronischen Verlauf haben, d.h. im Lebenslauf immer wieder auftreten können [5]. Die wichtigste Haltung im Umgang mit chronischen Problemen ist Akzeptanz [6], also die innere Annahme des unerwünschten Leidens. Um die Patienten im langfristig adaptiven Umgang mit der Krankheit zu unterstützen, setzen wir uns intensiv mit dem anspruchsvollen Prozess der Akzeptanz auseinander. Diese beinhaltet immer einen passiven Teil der Resignation wie auch die aktive Anforderung, mit dieser unerwünschten Last ein sinnvolles Leben zu führen. Wir definieren Akzeptanz daher auch als «aktive Resignation" [7]. Diese nimmt in unseren Behandlungen einen zentralen Stellenwert ein.

\section{Teil 2: Implementierung und Evaluation}

Da das Hohenegg-Kontextmodell die Perspektive des Patienten und seine Bedürfnisse nach tragfähigen, transparenten Beziehungen, nach fachlicher Expertise und sein besseres Verständnis für die therapeutischen Prozesse ins Zentrum rückt, haben wir über die medizinischen Professionen hinweg alle therapeutischen Angebote neu überdacht und mittels einer Methodenintegration besser aufeinander abgestimmt. Dabei kommt dem breit abgestützten, interdisziplinären Assessment eine besondere Bedeutung zu. Darauf basierend findet eine zielorientierte Behandlungsplanung statt, in welchem wir die aktive Beteiligung der Patienten im Sinne eines "shared decision making" aktiv fördern. Die wissenschaftliche Evaluation des Modelles, die wir acht Monate nach dem Start der Implementierung durchgeführt haben, zeigt eine erfreuliche Akzeptanz des neuen Modelles sowohl bei Patienten wie auch den Mitarbeitenden

\section{Existentielle Angst und Vertrauen}

Patienten, die in eine psychiatrische Klinik eingetreten sind, befinden sich in einem bedrohlichen Zustand des Kontrollverlustes und der existenziellen Angst. Die wichtigste Aufgabe der behandelnden Klinik besteht darin, durch den Aufbau von Vertrauen diese Existenzangst abzubauen.

\section{Basis des Hohenegg-Kontextmodells}

Das Hohenegg-Kontextmodell, so nennen wir unsere neue therapeutische Grundlage, bezieht sich auf den amerikanischen Psychologen und Psychotherapieforscher Bruce Wampold und wurde auf unsere Ansprüche adaptiert. Wampold ging insbesondere der Frage nach, welche Faktoren für den Therapieerfolg in Psychotherapien ausschlaggebend sind. Die Resultate können in folgender Weise zusammengefasst werden [8]: Es ist weder die fachliche Kompetenz noch die Methode des Therapeuten, die wesentlich über den Behandlungserfolg entscheidet. Der Erfolgsfaktor ist seine Fähigkeit, mit einem Patienten eine aus dessen Wahrnehmung gute Arbeitsallianz aufzubauen. Auf den Punkt gebracht sind dabei folgende drei Aspekte massgebend:

- Die Qualität der therapeutischen Beziehung aus Sicht des Patienten

- Die Expertise des Therapeuten aus Sicht des Patienten

- Die Plausibilität des Therapiemodells aus Sicht des Patienten

Anders gesagt: Es entscheidet nicht allein der objektive Inhalt der psychotherapeutischen Intervention über den Erfolg, sondern auch der Kontext - die aus Patientenperspektive überzeugende Art der Umsetzung. Im Grundsatz geht es um die Frage, wie das Patientenvertrauen gewonnen und verankert werden kann.

Bei der stationären Behandlung gehen wir davon aus, dass die Erkenntnisse von Wampold für die einzelnen therapeutischen Interaktionen gültig sind. Daher und im Wissen um die zentrale Bedeutung des Patientenvertrauens haben wir die therapeutischen Angebote und Prozesse unserer Klinik zweckmässiger strukturiert. Hierbei wollten wir die folgenden drei Aspekte des Patientenvertrauens fördern:

\section{Personales Vertrauen - therapeutische Beziehung, Kontinuität}

Wir versuchen möglichst übersichtliche, stabile und verlässliche therapeutische Beziehungen anzubieten: Jedem Patienten wird ein erfahrener, hochqualifizierter Facharzt oder Fachpsychologe zugeordnet, der für die gesamte medizinische Behandlung vom Zeitpunkt 
des Eintritts bis zum Austritt verantwortlich ist. In gleicher Weise ist die Pflege durch ein Bezugspflege-System individuell und transparent organisiert. Die Spezialtherapeutinnen sind ebenso klar zugeordnet, so dass sich der Patient an einer kleinen Zahl von definierten therapeutischen Bezugspersonen orientieren kann. Dank diesem System behält der fallverantwortliche Facharzt/Psychologe den Überblick über den gesamten Behandlungsverlauf und ist kompetente Ansprechperson und Koordinator für die Anliegen der externen Zuweiser.

Grundsätzlich findet zwischen den einzelnen therapeutischen Disziplinen ein reger fachlicher Austausch statt, der das gegenseitige Verständnis intensiviert. Die Ärzte und Psychologen wurden neu einem/einer der drei Patientenhäuser/Stationen zugeordnet, was zu einer intensiveren Kommunikation mit den Pflegefachpersonen geführt hat. Gegenseitiges Vertrauen wird jedoch nicht nur durch die einzelnen Fachpersonen, sondern durch die gesamte Arbeitsatmosphäre in unserer Klinik geschaffen.

\section{Krankheitsbezogenes Vertrauen - Expertise}

Wir arbeiten ausschliesslich mit hochqualifizierten Fachpersonen, die ein hohes Mass an Expertise mitbringen. In den von der Klinik festgelegten Schwerpunkten optimieren unsere Fachkräfte die therapeutischen Prozesse kontinuierlich. Dabei orientieren sie sich konsequent an den aktuell gültigen fachlichen Leitlinien. Die Patienten werden durch unsere Fachexperten gründlich und individuell über ihre Erkrankung informiert. Die für den erfolgreichen Umgang mit psychischen Problemen wichtigen Fragen zur inneren Haltung, wie solchen zur Akzeptanz von Leiden oder zur Bedeutung von Ressourcen [9], werden in

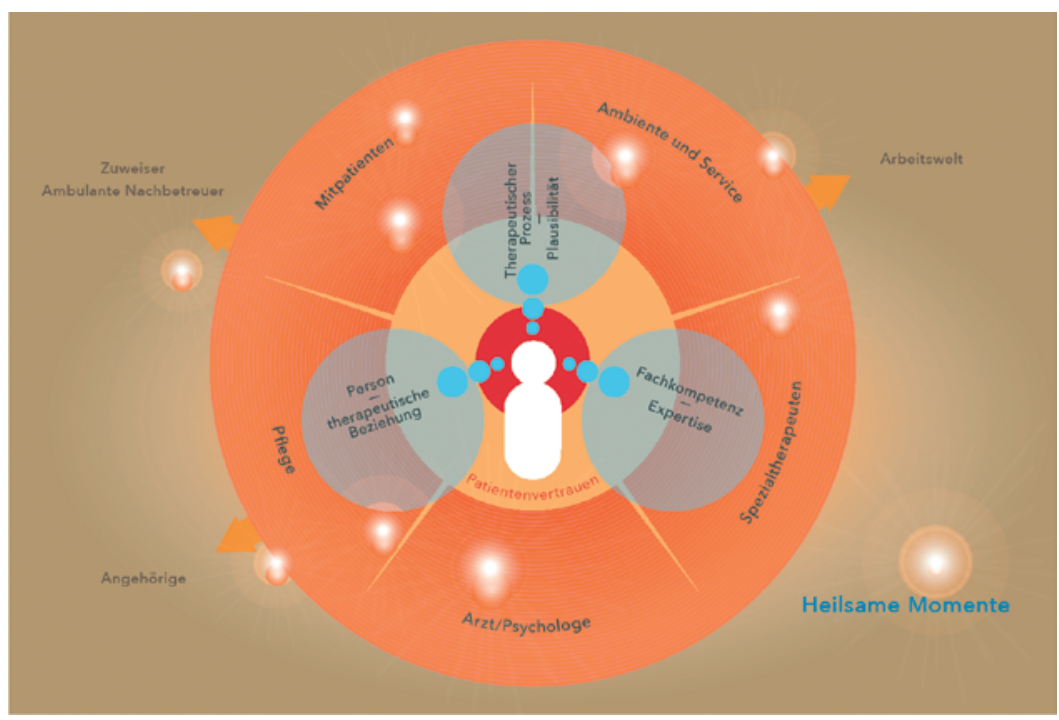

Abbildung 1: Das Hohenegg-Kontextmodell. ärztlich geleiteten Patientengruppen intensiv bearbeitet.

Zudem bieten wir auch verschiedene störungsspezifische Gruppen an, in denen der interaktive Austausch zwischen den betroffenen Patienten und unseren Fachärzten gefördert wird. Mittels des Einsatzes von neuen Medien, wie z.B. des Youtube-Kanals «Sprechstunde Psychotherapie» sind die vermittelten Inhalte jederzeit abrufbar. Zudem bauen wir unterschiedliche Informationsmittel (Flyer, Bücher oder Audio-CDs) auf, um die Informationsbedürfnisse unserer Patienten bestmöglich abzudecken.

\section{Prozessuales Vertrauen - Plausibilität}

Nach dem Eintritt wird der Patient darüber informiert, wie wir psychische Krankheit und Gesundheit verstehen und in welcher Weise wir während des Aufenthalts und danach seine psychische Gesundheit fördern wollen. Die einzelnen Therapieziele während der stationären Phasen sind auf ein integriertes Behandlungsmodell bezogen, das die verschiedenen Anliegen miteinander in Beziehung setzt. Sie sind allen Behandlern bekannt und werden von allen therapeutisch Tätigen mitgetragen. Die erwähnte Information geschieht interaktiv in einfacher, überzeugender und auf Bildsprache basierender Art. Deren Vermittlung bezieht Ärzte, Psychologen, Pflegefachpersonen, Spezialtherapeuten aber auch schon länger in der Klinik behandelte Patienten gleichermassen mit ein.

Wir legen sehr grosses Gewicht auf eine hohe Transparenz und Kooperation bei der Verschreibung von Psychopharmaka. Neben der Information über Wirkweise und Nebenwirkungen der Medikamente werden alle Patienten vertieft über die zentrale Bedeutung ihrer persönlichen Einstellung zur Einnahme von Psychopharmaka hingewiesen, welche durch die aktuellen Resultate der Placebo-Forschung im Bereich der Antidepressiva [10] eindrücklich belegt ist.

\section{Die Wirkmechanismen des Modelles}

Wir verstehen die gesamte Behandlung psychischer Erkrankungen als einen Prozess, in dessen Zentrum der Patient und dessen Erwartungen stehen. Auf dem Boden seines Vertrauens in die Kompetenz unserer Klinik kann der Patient seine Existenzangst und die Sorge, dass es «nicht mehr besser wird» abbauen. Mit abnehmender Angst und zunehmender Hoffnung kann sich der Patient wieder mutiger seinen Problemen stellen. So wachsen Zuversicht und Selbstvertrauen, welche die Grundlage für neue heilsame Momente sind. Diese besonders wirksamen Momente ereignen sich sowohl in den dafür vorbereiteten therapeutischen Gefässen der Psychotherapie, der Pharmakotherapie und den non- 
verbalen Therapien, können aber beispielsweise auch in Interaktionen mit Mitpatienten oder als bewusstes achtsames Naturerlebnis im Klinikareal geschehen. Dies basiert auf einem Verständnis, dass sich psychische Gesundung nicht in einem linearen Prozess der Anhäufung guter therapeutischer Interaktionen entwickelt, sondern meist in sprunghaften Entwicklungen erfolgt, d.h. in therapeutischen Momenten, in denen etwas Neues erkannt und erlebt wird.

\section{Einbezug der Aussenwelt im Hohenegg-Kontextmodell}

Unser Kontextmodell legt grosses Gewicht auf durchlässige Aussengrenzen. Die Klinik ist ein Ort, an den sich der Patient zurückziehen und Ruhe finden kann. Wir berücksichtigen aber auch sein zukunftsorientiertes reales Umfeld wie Angehörige, Arbeitswelt, Wohnsituation sowie die Vor- und Nachbehandlung.

Die Angehörigen werden aktiv in die individuellen Therapien einbezogen, u.a. indem wir monatliche Foren durchführen, welche Angehörigen und nahen Bezugspersonen unserer Patienten gezielte Unterstützung bieten. Die enge Zusammenarbeit mit verschiedenen Berufscoaches während der stationären wie auch der poststationären Behandlungsphase in unserem Ambulanten Zentrum in Zürich (AZZ) dient der Verbesserung der Reintegration unserer Patienten in ihre Berufswelt. Wir arbeiten zudem mit einem Netzwerk von ambulant tätigen Psychiatern, Psychologen, Spezialtherapeuten und Coaches zusammen, die sich in Intervisionsgruppen regelmässig treffen, um die Zuweisungs- und Nachbehandlungsprozesse zu optimieren.

\section{Zusammenfassung}

Wir verstehen die stationäre Behandlung psychisch erkrankter Menschen als einen interaktiven, nicht-linearen Prozess, in dem vertrauensbildende Begegnungen heilsame Momente fördern.

Das Hohenegg-Kontextmodell legt dabei auf zwei Dimensionen ein besonderes Gewicht:

- Innerer Kontext: Die aus Sicht des Patienten hochstehende Qualität der therapeutischen Beziehung sowie fachlich überzeugende und verständliche therapeutische Prozesse.

- Äusserer Kontext: Der reale Lebenskontext des Patienten (soziale Einbettung, Arbeitssituation und ambulantes Betreuungsnetz), der stark beachtet wird, um eine optimale Nachhaltigkeit der Behandlung zu fördern.

In unserem Modell entwickelt sich die sogenannte «evidence based medicine» zu einer «evidence informed medicine» [11] weiter: Das vorhandene medizinischtherapeutische Expertenwissen wird in bestmöglicher Weise auf die individuelle Situation übertragen und dem Patienten verständlich und nachvollziehbar vermittelt, um diesen zu ermächtigen und zu befähigen, die für ihn jeweils richtigen therapeutischen Entscheidungen zu fällen.

\section{Literatur}

1 Buber M (1983) Ich und Du. Lambert Schneider, Heidelberg.

2 Cassell EJ (1982) The nature of suffering and the goals of medicine. NEJM, 306; 639-45.

3 Huber M, Knottnerus JA, Green L, van der Horst H, Jadad AR, Kromhout D, et al. How should we define health? $2011 \mathrm{Jul}$ 26;343:d4163. doi: 10.1136/bmj.d4163.

4 Antonovsky A (1987) Unraveling the mystery of health: How people manage stress and stay well. Jossey-Bass, Chicago.

5 Westen D, Morrison K (2001) A multidimensional meta-analysis of treatments for depression, panic, and generalized anxiety disorder: an empirical examination of the status of empirically supported therapies. J Consult Clin Psychol, 69(6);875-99.

6 Hayes SC, Strosahl KD, Wilson KG (2011) Acceptance and commitment therapy: The process and practice of mindful change. Guilford Press, New York.

7 Jaspers K (1919) Psychologie der Weltanschauungen. Springer, Berlin.

8 Wampold B, Imel ZE (2015) The great psychotherapy debate, Routledge, New York.

9 Hobfol SE (1989) Conservation of resources: A new attempt at conceptualizing stress. Am Psychol,44;513-24.

10 Kirsch I, Deacon BJ, Huedo-Medina TB, Scoboria A, Moore TJ (2008) Initial severity and antidepressant benefits: A meta-analysis of data submitted to the food and drug administration. Plos Med, 5(2):e45 doi:101371/ journal.pmed. 0050045.

11 Miles A, Loughlin M (2011) Models in the balance: evidence-based medicine versus evidence-informed individualized care. J Eval Clin Pract, 17; 531-6.

Bildnachweis

(c) Martina Ott 